\title{
Chemoprophylaxis and HAART Therapy in Botswana M Essex*
}

Address: Department of Immunology and Infectious Diseases, Harvard School of Public Health, Boston, MA 02115 USA

Email: M Essex* - messex@hsph.harvard.edu

* Corresponding author $\ddagger$ Presenting author

from 2005 International Meeting of The Institute of Human Virology

Baltimore, USA, 29 August - 2 September 2005

Published: 8 December 2005

Retrovirology 2005, 2(SuppI I):SI24 doi:I0.II86/I742-4690-2-SI-SI24

Botswana has one of the highest rates of HIV infection in the world, with $37.4 \%$ of adults infected in 2003 according to UNAIDS. To address the problems of widespread infections, the government of Botswana instituted aggressive programs for the use of antiretroviral (ARV) drugs in both chemoprophylaxis for pregnant women and HAART therapy for treatment of clinical AIDS. At present, about $25 \%$ of AIDS patients receive HAART, and about $40 \%$ of HIV positive pregnant women receive chemoprophylaxis. After the country adopted an "opt out" policy for HIV testing in early 2005 , these numbers are rapidly rising. Both the chemoprophylaxis regimen and the first line regimen for AIDS, include nevirapine (NVP). This is likely to cause problems with drug resistance when mothers with young infants need therapy, as about half of the mothers who receive NVP during labor reveal genotypic resistance when analyzed. In a recent trial we showed that NVP given only to newborn infants is as effective as when given to the mother during labor and the newborn infant, at least when used on a background of zidovudine (ZDV). This may provide a mechanism to avoid the establishment of NVP-resistance in HIV-positive mothers. HIV-1C, the subtype of southern Africa, shows higher rates of NVP-resistance as compared to HIV-1A or HIV-1D.

Despite the potential for high levels of resistance, and advanced stages of disease, AIDS patients were very successfully treated with HAART. With initiation of drugs at median plasma viral loads of about 400,000 and median CD4 counts below 100, 87\% of patients treated with ZDV+ 3TC+ NVP had undetectable RNA at 24 weeks after treatment, and $79 \%$ had undetectable RNA at 48 weeks. CD 4 numbers increased by 149 at 24 weeks and 204 by 48 weeks. ZDV and 3TC containing regimens gave lower rates of viral resistance and less toxicity than regimens containing DDI and D4T. 